Public Abstract

First Name:Joseph

Middle Name:Preston

Last Name:Driewer

Adviser's First Name:H. Harold

Adviser's Last Name:Li

Co-Adviser's First Name:Sudarshan

Co-Adviser's Last Name:Loyalka

Graduation Term:SS 2011

Department:Nuclear Engineering

Degree:PhD

\title{
Title:DOSIMETRIC PROPERTIES AND RADIATION HARDNESS OF THE STORAGE PHOSPHOR EUROPIUM DOPED POTASSIUM CHLORIDE FOR RADIATION THERAPY DOSIMETRY
}

Multidimensional reusable dosimeters are extremely important for characterizing the complex dose distributions associated with modern radiation therapy techniques, such as intensity modulated radiation therapy (IMRT). In particular, reusability provides medical physicists a degree of confidence in dosimetric measurements through the acquisition of benchmarking datasets and through long-term, repeated use and performance monitoring. Yet a dosimeter that has all of the properties desired for radiation therapy use, such as reusability, high spatial resolution, and water-equivalence, is not currently available. The results of this dissertation demonstrate that the reusable storage phosphor europium doped potassium chloride, $\mathrm{KCl}: E u 2+$, has the potential to significantly advance the state-of-the-art in radiation therapy dosimetry.

Prototype chip and panel dosimeters were fabricated according to well-developed materials science processes. Dosimetric properties were studied by reading photostimulated luminescence (PSL) after irradiation with a laboratory optical system. Sensitivity was independent of dose rate and beam energy for commonly used therapy beams. Over-response to low-energy scattered photons was comparable to conventional radiographic film and reduced with lead foils. No significant changes in the PSL process were detected up to 5000 Gy dose history, indicating that the material could be reused up to 2500 times at 2 Gy per use, as in, for example, patient-specific IMRT quality assurance. Investigations of KCl:Eu2+ panel dosimeters show that sub-millimeter spatial resolution is achievable and that micron-scale dosimeters have a water-equivalent dose response with sufficient sensitivity over clinically relevant dose ranges.

In conclusion, $\mathrm{KCl}: \mathrm{Eu} 2+$ is demonstrated to have many desirable properties for radiation therapy dosimetry. This study provides a practical and theoretical knowledge base that advances and supports future $\mathrm{KCl}:$ Eu2+ dosimetry research. 